\title{
Patient-Reported Outcome Monitoring in Brain Tumour Patients: Benefits and Requirements
}

\author{
Lisa M. Wintner, Johannes M. Giesinger and Bernhard Holzner \\ Innsbruck Medical University \\ Austria
}

\section{Introduction}

In 2008 within the European Union brain cancer and cancer of the nervous system ranked number 15 within the 20 most diagnosed cancer types (Ferlay et al., 2010). Compared to the five most frequent cancer types brain cancer seems to have a small-sized incidence-rate, but the mortality rate for this type of cancer is much higher than those of most high-incidencecancers (except lung cancer). Though brain cancer is only seldom diagnosed, its negative impact on patients health status and well-being should not be disregarded, as survival prognosis is poor and deteriorated health-related quality of life (QOL) is common (Taphoorn et al., 2010). Treatment options comprise surgery, radiation, chemotherapy and supplementary medical therapies. Brain cancer is characterised by a large variety of tumour types and a distinction is made between primary tumours arising directly from brain tissue and secondary tumours, which are brain metastases from other malignant diseases. This differentiation is often difficult to make, though, it is of special importance since the best possible treatment e.g. for glioblastoma strongly differs from those for brain metastases (Campos et al., 2009) and inadequate intervention jeopardises patients' QOL.

Survival expectancy is strongly related to the WHO grade of the tumour and varies vigorously. Grade I tumours may not shorten life expectancy, grade II tumours are associated with a survival time of 2-10 years, grade III tumours with a survival time of 2-3 years and grade IV tumours with a survival time of only 9-12 months (Reardon \& Wen, 2006). Not only the tumour itself, but also treatment-related side effects and adverse events caused by supportive medication (e.g. steroids and antiepileptic drugs (Sizoo et al., 2010)), confront the patient with a high level of physical and psychosocial burden. Brain tumour patients are affected by a diversity of tumour symptoms, which might be caused by increased intracranial pressure (e.g. headache, anorexia, seizures, nausea, vomiting, sleeping longer at night, drowsiness, napping during the day). Further physical impairments may occur due to focal neurological deficits (e.g. motor deficit, aphasia, visual field defects) and even more burdensome both for patients and care-givers might be symptoms like personality changes, decrease in mental capacity and concentration, mood disturbances, cognitive dysfunction, fatigue and anxiety (Heimans \& Taphoorn, 2002; Sizoo et al., 2010). These symptoms were found to have a negative impact on both patients' and their carers' overall QOL if compared to general population (Janda et al., 2007). As brain tumour patients cannot be cured in most cases, the treatment has to focus on maintaining QOL, to make the patient's remaining life time as enjoyable as possible. 


\section{Patient-reported outcomes}

A short and concise definition of patient-reported outcomes (PROs) is presented by the Food and Drug Administration: "A PRO is a measurement of any aspect of a patient's health status that comes directly from the patient (i.e., without the interpretation of the patient's responses by a physician or anyone else)."(U.S. Food and drug administration (FDA), 2006). Therefore, PROs allow the comprehensive evaluation of patients' perception of aspects of functioning and well-being in regard to their health status, disease, and its treatment. They comprise a variety of directly reported health-related issues: disease-related symptoms, treatment-related adverse events, functioning, well-being, health-related quality of life (QOL), perceptions about treatment, satisfaction with care and professional communication (Rothman et al., 2007). Commonly assessed PROs are e.g. QOL, depression, anxiety, pain, fatigue, gastrointestinal symptoms, social functioning, or perceived cognitive dysfunction. Thus, PROs are characterised by versatility in possible application, as they may be used for QOL evaluation both in clinical research and routine, for adverse event detection in drugsafety reports and medical product development, and for guidance in medical decision making.

Many studies already illustrate that PRO-data provides additional information, which is useful for medical decision-making and treatment choices. Two papers report the results from a study with newly diagnosed glioblastoma patients (Stupp et al., 2005; Taphoorn et al., 2005). Intensive treatment comprising radiotherapy and chemotherapy with temozolomide was compared to standard treatment in regard to survival and QOL. The combined treatment option was generally refused by clinicians, since they supposed the potential side-effects to outweigh the possible benefit of longer survival. PRO-assessment at baseline, during and after treatment showed that patients with combined radio- and temozolomide chemotherapy survived longer than patients with radiotherapy alone, without additional impairments of self-reported QOL.

Marino et al. (2008) investigated QOL and overall survival in breast cancer patients receiving conventional chemotherapy or high-dose chemotherapy. PRO-data revealed that high-dose chemotherapy caused severe impairments in functioning scales and considerably high ailments due to nausea, fatigue and pain during active treatment. Furthermore patients' QOL recovered more slowly when high-dose treatment was administered and QOL remained stable at a lower level than QOL of patients with standard treatment. This additional QOL impairment due to high-dose therapy was not associated with longer overall survival.

A study conducted by Press et al. (2001) and Ganz et al. (2003) compared treatment options for patients with early Hodgkin's disease. The patient group who received chemotherapy before standard treatment (subtotal lymphoid irradiation, SLI) did not suffer from higher toxicity, had a higher failure-free survival and fewer relapses or deaths than patients receiving only SLI. Though, these benefits in respect to clinical outcomes were associated to a greater severity of symptoms, more fatigue and poorer QOL six months after baseline. Nevertheless, it must be kept in mind that treatment with chemotherapy lasted twice as long as standard treatment and therefore time for recovery was longer in SLI patients. At the one year follow-up QOL-scores returned to baseline levels in both treatment groups.

Individual patients as well as health care providers can benefit from PROs, as these may not only support patients in selecting an available treatment, but also enhance the general understanding of the disease and improve its treatment and the management of treatment- 
related side-effects. Additionally, PROs may alleviate the decisional hierarchy between clinicians and patients by involving both sides into the formulation of important health and QOL domains, which are considered to need clinical attention (Snyder et al., 2010). In this way, medical issues do not longer suppress QOL domains. Besides, by giving patients' the possibility to contribute to the choice of medical treatment, their anxiety and depression can be reduced and their QOL improved (Arora, 2003).

A high percentage of clinicians support the assessment of PRO-data, but only one third of them has knowledge about PRO-instruments and procedures for data collection (Bossola et al., 2010). This lack of understanding, of what PROs exactly are and what they can contribute in daily routine, could be one reason that clinicians see their authority questioned and refuse to work with PROs. However, PROs do not intent to compete with direct physician-patient communication or to replace clinicians' expert opinion. PROs rather want to improve the ability to identify and detect specific impairments and symptoms including psychological problems. The integration of patients' individual perspective may also improve multi-professional cooperation and the combination of different medical and psychological treatment options to a patient-tailored and comprehensive therapeutic concept. Many clinicians argue that the use of PROs would take too much time, but it is reported that follow-up appointments did not or only 0.5 to 2.7 minutes prolong due to PRO-assessment (Frost et al., 2007).

The additional value of PRO-data in clinical routine was shown in some randomisedcontrolled trials. The prescription of pain medication in a mixed sample of cancer patients differed significantly between patients who completed a pain-self-rating and patients who did not. Prescriptions for patients who provided a self-report changed more often, both with increase and decrease. In patients without self-reports prescriptions changed less frequently and only with an increase (Trowbridge et al., 1997). In another study, clinical staff responsible for lung cancer patients was demonstrated how to read PRO-data and they were encouraged to use reports of these data to detect domains, which could guide the discussion about QOL with the patient. Patients whose clinicians used PRO-data reported more domains discussed in regard to QOL within the consultation (Taenzer et al., 2000). In patients with various cancer diagnoses undergoing palliative chemotherapy, the use of PRO-data before the follow-up visits prior to each chemotherapy cycle had a number of significant effects: communication about QOL issues improved, unobvious domains like social functioning and fatigue were more often discussed and patients' felt to a larger extent emotionally supported. Clinicians showed a tendency to be more responsive to problems in unobvious QOL-domains. The duration of the follow-up visit was not prolonged by the use of PRO-data (Detmar et al., 2002a). Another population of cancer patients with different diagnoses receiving chemotherapy gave evidence that the explicit use of PRO-data can have a positive impact on patients' QOL and emotional well-being (Velikova et al., 2004). Of these patients whose PRO-data was explicitly used, the vast majority rated the questionnaire to be useful to communicate personal feelings to the clinician (Velikova et al., 2010).

Although studies have shown that PROs can be useful for enhancing communication in clinical routine, there are also studies that do not report a beneficial impact of PROs. Some studies did not find any effect of the usage of PRO-data on global QOL of patients (Drury et al., 2000; McLachlan et al., 2001; Detmar et al., 2002a). A randomized-controlled trial conducted by Rosenbloom et al. (2007) provided PRO-data and results of structured interviews of advanced cancer patients undergoing chemotherapy to their nursing staff. No 
effect of the mere provision of PRO-data on clinical management strategies, satisfaction or QOL was found. The authors suggest that the communication of PRO-data is only useful, if oncology staff is also provided with concrete information about treatment and care strategies for certain QOL-impairments. This criticism concerns all of the above mentioned studies investigating PROs in clinical routine, as none of them gives any specific treatment advice to clinicians (Taenzer et al., 2000; McLachlan et al., 2001; Detmar et al., 2002a; Velikova et al., 2004; Velikova et al., 2010).

Osoba (2007) emphasizes that PROs can be useful at several stages of patient-management, especially when they are conducted in parallel to conventional medical procedures. Besides gathering information about patients' medical history, physical exams, laboratory and imaging tests, treatment and follow-up, PROs may be used to support differential diagnosis and to monitor patients' progress. Similar to these steps, which are associated with several courses of action, also PRO-data has to be associated with guidelines for clinicians, which interventions can be set, if impaired QOL-domains are detected. Clinicians have to be trained in PRO-data interpretation and in possibilities for QOL-focused support. By analogy, it would seem bizarre to argue that blood level analysis is useless, because clinicians do not adjust treatment in regard to these characteristic values due to insufficient knowledge about how to use them. Exactly this is a problem with PRO-data: most studies do not evaluate specific interventions imposed after PRO-data analysis, but only the collection of PROs. Some studies tell clinicians to use PRO-data within patient-clinician communication, but do not guide them how to use this data. Therefore the study results might depend on the open-mindedness, prior experience and the expertise about PRO-use of the clinician. Furthermore, some patients might already benefit from the mere collection of PRO-data and others might need focused intervention.

Regarding the studies that did not find any positive effect of PRO-data, some further limitations have to be taken into account. There was only a low compliance of clinicians' to pay attention to PRO-data and to use them regularly. Just a third of all possible offered interventions were used by patients and physical procedures were more often accepted than psychological consulting (McLachlan et al., 2001). Furthermore, ceiling effects may have disguised existing effects of PRO-data usage on patients' satisfaction (Taenzer et al., 2000; Detmar et al., 2002a).

Even if the results of randomized-controlled trials concerning the usage of PRO-data are mixed, there is evidence that PROs can enrich patient-clinician communication and in this way also influence patients' QOL. PRO-completion seems not to impose an intolerable burden to patients and many studies report high acceptance of PROs - conducted as paperpencil or more sophisticated via a software program - even in sick patients (Mullen et al., 2004; Bush et al., 2005).

The acceptance of PROs by patients is usually very good, as they are capable to bridge the gap between favoured discussions on QOL-domains with their clinician and patients' hesitation to initiate these discussions themselves. Detmar et al. (2000) reported that QOL domains differ in regard to how often patients wish their clinician to start a conversation on a certain issue. Approximately $25-29 \%$ of patients wish their clinician to ask them about their emotional functioning, daily activities and familial issues. About $37 \%$ of patients expect their clinician to address the topic of social functioning. Hereby PROs can successfully contribute to satisfaction of clinicians and patients, as clinicians attain a deeper insight in patient's needs and may properly address relevant topics and patients do not 
need to answer a variety of possibly irrelevant questions during the face-to-face consultation. PROs may also sharpen clinicians' awareness for psychological, social and spiritual functioning and diminish the domination of physical functioning in patient-doctor conversation (Rodriguez et al., 2010).

\subsection{PRO instruments}

For PRO-assessment in cancer patients, an extendable generic cancer core-questionnaire can be administered, which might be supplemented by disease-specific questionnaire-modules. Widely used, validated and reliable cancer-targeted instruments for instance are the European Organization for Research and Treatment of Cancer core questionnaire (EORTC QLQ-C30) and the Functional Assessment of Cancer Therapy general version (FACT-G). To both of these instruments a brain cancer-specific module can be added (QLQ-BN20 for the EORTC QLQ-C30 and FACT-Br and the FACT-Br Symptom Index (FBrSI) for the FACT-G). Table 1 gives an overview of the questionnaire characteristics.

\subsubsection{EORTC QLQ-C30 and QLQ-BN20}

The EORTC QLQ-C30 was originally developed to evaluate QOL in cancer patients participating in clinical trials (Aaronson et al., 1993) and is a nowadays widely used and internationally validated cancer-targeted PRO-instrument. The core questionnaire consists of thirty questions and is the basic part of the QOL-instrument, which can be expanded by additional elements. These supplementary modules focus on diagnosis-specific symptoms and impairments. Altogether, the QLQ-C30 comprises nine scales and six single item symptoms. Five scales concern various functioning domains (physical, role, social, emotional and cognitive functioning), one is a scale for global QOL, and three are symptom scales (pain, fatigue, nausea/vomiting). Except for the two questions about general QOL every question needs to be rated on a likert scale from "not at all", "a little", "quite a bit" to "very much". The overall QOL scale asks the patients to grade how they experienced their general QOL and physical condition between 0 "very poor" and 6 "excellent". With the exception of the physical functioning scale, all questions use a time frame of one week before the examination date.

The EORTC Brain Cancer Module (QLQ-BN20) contains 20 items and focuses on symptoms that particularly concern brain cancer patients undergoing chemotherapy or radiotherapy. In this way, both disease symptoms and treatment toxicities are included in the item list (Sprangers et al., 1998). The QLQ-BN20 is divided into four scales (future uncertainty, visual disorder, communication deficit, motor dysfunction) and seven single items (headache, seizure, drowsiness, hair loss, itching, weakness of both legs, difficulty controlling bladder function) (Osoba et al., 2000).

\subsubsection{FACT-G and FACT-Br/FBrSI}

The Functional Assessment of Chronic Illness Therapy (FACIT) measurement system provides questionnaires concerning a variety of chronic illnesses and their conditions, for instance cancer, HIV/AIDS, and multiple sclerosis. Within the FACIT the FACT-G questionnaire is a cancer-specific instrument for PRO-assessment (Webster et al., 2003). Nowadays the fourth version of the FACT-G is widely used and comprises 27 items grouped into four primary QOL domains (physical well-being, social/family well-being, emotional well-being, and functional well-being). The patient has to rate each item on a five- 
point Likert-scale between "not at all", "a little bit", "somewhat", "quite a bit" and "very much" in relation to symptom severity during the last week. The FACT-G was examined concerning its ease of administration, brevity, reliability, validity and responsiveness to clinical change and was found to fit all the stipulated requirements (Cella et al., 1993). The questionnaire's validity was also tested for a variety of language versions (Sanchez et al., 2011).

The brain cancer-specific FACT-Br module consists of 23 items and explores how patients perceive their cognitive, neurological, sensory and psychological functioning and the impact of changes in these domains on their daily living. Additionally, the FACT-Br Symptom Index (FBrSI) is available for symptom rating of brain tumour patients. Both the FACT-Br and FBrSI proved to be reliable, valid and responsive to change (Nickolov et al., 2005).

\begin{tabular}{|c|c|c|}
\hline instrument & scales & items \\
\hline $\begin{array}{l}\text { FACT-G } \\
\text { Functional Assessment } \\
\text { of Cancer Therapy- } \\
\text { General }\end{array}$ & $\begin{array}{ll}\text { - } & \text { physical well-being } \\
\text { - } & \text { social/family well-being } \\
\text { - } & \text { emotional well-being } \\
\text { - } & \text { functional well-being }\end{array}$ & 27 \\
\hline $\begin{array}{l}\text { FACT-Br } \\
\text { Functional Assessment } \\
\text { of Cancer Therapy-Brain } \\
\text { Module }\end{array}$ & $\begin{array}{ll}- & \text { cognitive functioning } \\
- & \text { neurological functioning } \\
- & \text { sensory functioning } \\
- & \text { psychological functioning } \\
- & \text { impact of changes in functioning on daily } \\
& \text { living. }\end{array}$ & 23 \\
\hline $\begin{array}{l}\text { FBrSI } \\
\text { FACT-Br Symptom } \\
\text { Index }\end{array}$ & brain tumour specific symptom list & 15 \\
\hline $\begin{array}{l}\text { EORTC QLQ-C30 } \\
\text { European Organisation } \\
\text { for Research and } \\
\text { Treatment of Cancer } \\
\text { Quality of Life } \\
\text { Questionnaire Core } 30\end{array}$ & $\begin{array}{ll}\text { - } & \text { functioning (physical, role, social, emotional } \\
\text { and cognitive) } \\
\text { - } & \text { global QOL } \\
\text { - } & \text { pain, fatigue, nausea/vomiting } \\
\text { - } & \text { dyspnoea, sleeping disturbances, appetite } \\
\text { loss, constipation, diarrhoea and financial } \\
\text { impact }\end{array}$ & 30 \\
\hline $\begin{array}{l}\text { EORTC QLQ-BN20 } \\
\text { European Organisation } \\
\text { for Research and } \\
\text { Treatment of Cancer } \\
\text { Quality of Life Brain } \\
\text { Module }\end{array}$ & $\begin{array}{ll}- & \text { future uncertainty } \\
\text { - } & \text { visual disorder } \\
\text { - } & \text { communication deficit } \\
\text { - } & \text { motor dysfunction) } \\
\text { - } & \text { headache, seizure, drowsiness, hair loss, } \\
& \text { itching, weakness of both legs, difficulty } \\
& \text { controlling bladder function }\end{array}$ & 20 \\
\hline
\end{tabular}

All instruments have been extensively validated.

All instruments use a recall period of one week.

Table 1.Commonly used PRO-instruments for cancer patients with brain modules available 


\subsection{The value of proxy-reported outcomes}

A common symptom burden in brain cancer patients are cognitive impairments, which may be caused by the disease itself or anti-cancer treatment. Although PROs are the method of choice for QOL-assessment, this method is not suitable for patients who suffer from deteriorated cognitive function. A large amount of patients is not able to complete PROinstruments due to serious malfunction in cognition, speech, vision, concentration or language. Even if these symptoms are perceived at a low level, PRO-assessment can be too burdensome for these patients and cause additional distress. This implies that the needs of a certain subgroup of patients are possibly not adequately met, because they are excluded from QOL-assessment and therefore also from intervention. Furthermore, the exclusion of these patients from longitudinal studies sugarcoat data and may produce compromising and misleading results (Sneeuw et al., 1997). Especially the subgroup of patients, who are not able to give information about their QOL themselves, might particularly be in need for intervention concerning impaired QOL-domains.

A complementary source of information about patients' QOL are therefore ratings from clinicians, nurses or significant others (spouses, children, family members or close friends). Normally, the usefulness of information provided by others on patients' QOL is investigated by analysing the agreement of outsiders' and patients' ratings. Therefore, proxies or health care providers have to complete the same PRO-instrument as the patient. The degree of agreement or correlation of patients' and others' answers reflects the reliability of this approach for QOL-data collection. Generally, proxy ratings tend to overestimate patients' symptom burden and underestimate their QOL, because proxies are in their ratings influenced by their own feelings and experiences of care-giving activities. Nevertheless, care-giving significant others are supposed to be a valuable source for information about patients' QOL, especially for concrete and observable domains (Addington-Hall \& Kalra, 2001). In brain cancer patients there was exact agreement between proxies and patients found in $60 \%$ of the questions of the EORTC QLQ-C30 and the module BN20. If one category of difference in either direction was allowed, even over $90 \%$ of approximate agreement was obtained. There were only low correlations between ratings of social functioning, nausea/vomiting, and pain. Over time, agreement worsened significantly for patients whose condition deteriorated since the baseline assessment. Additionally, disagreements in answers were more likely if patients were more physically and neurologically impaired or mentally confused (Sneeuw et al., 1997).

In brain cancer patients a moderate-to-high patient-proxy agreement for the Linear Analogue Self-Assessment (comprises questions concerning physical condition, social interactions, psychological effects of disease and personal relationships), for hope, confusion and sadness was reported (Hahn \& Dunn, 2003). Brown et al. (2008) report for patients with newly diagnosed high-grade glioma a moderate-to-high patient-proxy agreement at the FACT-Br questionnaire. They also indicate that agreement was influenced by patients' cognitive status. Low scores at the Folstein Mini-Mental State Examination were associated with a poorer agreement between proxies and patients in their rating of mood states. Giesinger et al. (2009a) report not only high discrepancies of patient-proxy rating for social functioning, dyspnoea and seizures, but also good agreement for the scales physical functioning, sleeping disturbances, appetite loss, constipation, financial impact and taste alterations (EORTC QLQ-C30 and BN20). They support the use of proxy-ratings for QOLassessment in brain cancer patients, but also critically note that general agreement is higher for physical symptoms than for psychosocial issues. 
All in all, patients and proxies exactly agreed in their answers to a high extent and variations mostly did not exceed more than one answer category (Sneeuw et al., 1997). Cognitive impairments seem to worsen agreement between patients and proxies (Sneeuw et al., 1997; Brown et al., 2008). More obvious physical symptoms show better agreement rates than psychosocial issues (Giesinger et al., 2009b). Although proxy-patient agreement deteriorates over time if patient's health and/or mental condition get worse, it still is reasonable to collect proxy-reported QOL-data for incorporating also the most ill patients in data-collection for intervention and research purposes.

\section{Electronic PRO-data capture}

Even though PRO assessments gain more and more importance in scientific research, e.g. concerning drug evaluation studies and label claim issues, they are not yet completely accepted as measurement method for adverse event detection, QOL-measurement and primary outcome in randomized controlled trials. Currently, many clinicians still consider clinician ratings' as being sufficient for adequate evaluation of patients' well-being and symptom burden (Basch, 2010). Patrick (2007) emphasizes the usefulness of PRO instruments, as they allow the capture of advantages and disadvantages of known or newly developed treatment options, which is valuable for choosing between therapies and for reasoning already chosen disease management strategies. One third of palliative cancer patients received adjusted treatment in respect to QOL improvement, but clinicians only focused on overall QOL instead of a variety of subscales and eclipsed QOL issues if patients showed tumour progression or increased treatment toxicity (Detmar et al., 2002b). This illustrates that specialized training for physicians is necessary to ladle the potential benefit of PRO-data in clinical routine.

Walker et al. (2003) report administrative failure as most important reason for missing data (72.2 percent) in PRO-data collection in patients suffering from malignant glioma. About 21.7 percent of patients did not fill out the PRO-instruments due to their very bad health condition and only 6.1 percent of patients refused to participate in PRO-assessments. The major problem of administrative failures in the study of Walker et al. (2003) can be traced to irregular administration time points of QOL PROs, too little explanation of instrument completion and missing specialized staff for QOL research who checks questionnaires for completeness. Such barriers can easily be overcome with the routine usage of electronic ePROs, which avoid a high percentage of missing data, as a standardized questionnaire explanation is integrated in the procedure and further questions are only displayed after all preceding questions have been answered.

\subsection{Requirements for ePRO and its implementation}

The implementation of ePRO in clinical routine requires some underlying circumstances, which enable effective QOL-evaluation. Firstly, there is a need for a comprehensive IT infrastructure that includes technical devices for data collection, software solutions for calculation and graphical illustration of results and network facilities for data-transmission, storage and backup. For data collection personal computers (PCs), laptops or personal digital assistants (PDAs) can be used. These devices differ in their feasibility according to patient-groups.

For outpatients a fixed PC-workstation can be useful, since these patients are supposed to be in a relatively good health condition and able to see the PC by themselves. Portable devices 
like laptops and PDAs seem to be more useful for inpatients, who are approached directly at the hospital bed. The IT infrastructure also should include a database for data storage and backup. A central database allows that clinicians from different wards have access to patients prior and latest QOL-assessments. A database connection can be established via a local area network (LAN) or Wifi (wireless fidelity, also WLAN, wireless local area network). Connection via LAN is more laborious as the study nurse needs to connect the tablet PC to the LAN with the purpose of preparing the patient list to who the questionnaire shall be administered. The tablet PC has to be disconnected, handed over to patients for bedside assessment and again connected to the LAN for uploading the collected information into the database.

A connection provided by WiFi is much more comfortable, as the database can be updated anytime without special constraints. Wifi is nowadays a common and inexpensive technology that is also practicable in a hospital setting. Connection via WiFi eases work for both data collectors and clinicians, as data is transferred instantly and the immediate access to patients' data is possible. Especially for home monitoring some additional requirements are added. If home monitoring is conducted via web-access, a feasible and user-friendly website with special security features is needed. Privacy of health information, data security and patients' safety need special attention when developing an online PRO-platform (Basch et al., 2007).

Secondly, clinicians and nurses need to be trained in two ways: on the one hand in device and software handling and on the other hand in choosing interventions according to PROdata. Acquaintance with technical equipment allows to answer most of patients' questions and to solve minor problems directly on site. Training in result interpretation is also necessary, as PRO-data is supposed to give information about patients' QOL and initiate thereby medical and/or psychosocial intervention. Although there are software solutions that offer easily interpretable graphs, precise instructions for interventions are still of crucial importance. Interpreting QOL-scores correctly is only the first step of successful ePRO-data use. The second step is to choose appropriate intervention strategies for QOL-management. Furthermore, a positive attitude of health care providers towards PRO-assessment is strongly necessary. Clinicians and nurses exert influence on patients and therefore also affect their compliance, since patients often orientate themselves by clinicians' suggestions. The vivid contribution of clinicians and nurses to assess and use PRO-data may also carry patients along and support the whole PRO-procedure in all stages.

\subsection{Feasibility}

Velikova et al. (1999) report a high acceptance of ePRO in a mixed sample of cancer patients. Patients were asked to complete a paper-pencil as well as an electronic version of the EORTC QLQ-C30, with an interval of three hours between the two assessments. More than half of the patients preferred the ePRO version to the traditional paper-pencil questionnaire, whilst each $24 \%$ of patients preferred the paper-pencil questionnaire or had no preference. Overall, the ePRO version was slightly quicker completed by patients, whereas older patients in general needed more time to complete the PRO-instruments, independent of the type of administration. The quality of collected QOL-data was excellent if electronic assessment was used, as no missing data occurred. Although Velikova et al. (1999) hypothesized that patients would rather refuse to take part in a study, which demands them to use a computer for data-collection, compliance rates were similar to those in traditional therapeutic clinical trials. 
The assessment of QOL by means of ePROs is also feasible in a clinical routine setting with a large number of mixed cancer patients attending the ward of an oncology clinic. Wright et al. (2003) conducted two feasibility studies, whose results underline that in comparison to a separate prospective patient sample (study 1), routine ePRO-assessment (study 2) reached higher compliance rates and therefore a better data-base for the description of changes over time. In both studies the majority of patients did not feel that the ePROs made their visit more difficult or temporally longer and only few patients said that they would refuse to complete ePROs as part of clinical routine. Most patients reported that the electronic equipment was easy to use and general feedback on ePROs was positive.

Carlson et al. (2001) used a computerized version of the EORTC QLQ-C30 that was already successfully utilized by Taenzer et al. (2000) for investigating the ePRO-based changes in lung cancer outpatient care. Already in this previous study (Taenzer et al., 2000) the QOLtool was shown to be feasible and effective in sensitizing clinicians for QOL-issues. In the study of Carlson et al. (2001), the effectiveness of ePROs was examined with a group of patients with extensive symptom burden, who attended a cancer pain and symptom control clinic. The majority of patients rated the computerized EORTC QLQ-C30 version to be easy in use and well understandable. Their satisfaction with the ePRO was high and with repeated use of ePRO they judged the use of computers more favourably. Clinical staff felt that the ePRO was appropriate for QOL-assessment and useful for guiding the interaction with the patient. All in all, both highly impaired patients and health care providers support the use of ePRO and confirmed their feasibility and usefulness.

\subsection{Symptom monitoring and screening}

Brain cancer patients are affected by a variety of severe clinical symptoms. Since the survival time in most brain cancer patients is rigorously limited, early and symptom-focused intervention is of paramount importance. PRO-data works in two ways concerning symptom management. On the one hand, QOL-evaluation reveals impaired domains, which need targeted intervention. On the other hand, regularly assessed PRO-data allows achieving a deeper understanding of the impact of symptom management on a variety of symptoms besides the aimed symptom (Ganz \& Goodwin, 2007). By way of example, there was the intention to improve physical functioning by pain reduction and PRO-data might show that other QOL-domains changed as well. PRO-assessments also capture unexpected developments and may illustrate interrelationships among QOL-domains (Ganz \& Goodwin, 2007).

Next to treatment-related symptoms, cancer patients have also to deal with psychosocial distress triggered by the disease itself and anti-cancer therapy. Since the inadequate or missing treatment of psychosocial distress may confront the patient with further impairments, as they might develop chronic mental disorders, early detection of psychosocial distress is necessary (Meraner et al., 2009). Effective screening for psychosocial distress can be performed by means of PRO-instruments like the Hospital Anxiety and Depression Scale (HADS) (Zigmond \& Snaith, 1983) or the General Health Questionnaire (GHQ-12) (Goldberg, 1972). Approximately 20-40 percent of cancer patients suffer from psychosocial distress and are therefore in need for referral to psychooncological treatment (Meraner et al., 2009). A study of Zabora et al. (2001) identified brain cancer patients to be highly endangered to perceive extensive psychosocial distress. Nearly 43 percent of brain cancer patients were tested positive with a distress screening by means of the Brief Symptom Inventory and ranked second after lung cancer with only tiny difference. For 
these patients, PROs contribute in the detection of needs for psychooncological treatment and therefore prevent further ailments.

As a screening tool is supposed to offer a quick possibility to check for impairments that require treatment, cut-off scores are of major importance. Additionally, the comparison to norm or reference values from general population or other cancer cohorts plays an essential role for the definition of impaired or normal QOL. For the interpretation of changes in PROscores it is necessary to know, which score-deviations reflect clinical meaningful changes. Statistically significant effects are easily to find if a high number of patients contributed in PRO-data collection, but not every significant effect also must be a subjectively meaningful difference (Cella \& Nowinski, 2002). To correctly interpret changes in PRO-scores, minimal important differences (MID) can be used. Jaeschke et al. (1989) described MID as the "smallest difference in score in the domain of interest which patients perceive as beneficial and which would mandate, in the absence of troublesome side effects and excessive cost, a change in patient's management" (as cited in Dubois et al., 2006, p 977). MIDs are diverse for PRO-instruments and may also differ between diagnoses and disease stages (Revicki et al., 2008). Furthermore, they vary in dependence on how good baseline scores were and if QOL increased or deteriorated (Farivar et al., 2004). In addition, it is necessary to know if a change in the QOL-score is associated to the patients' experience with a small, medium or large effect (Guyatt et al., 2002).

There are considerable differences in the MIDs, depending on whether individual or group scores are used for analysis. For the EORTC QLQ-C30 and the FACIT system MIDs for group levels have been established but these MIDs should not be used in the same way for individual score interpretation (Giesinger et al., 2009a). On average unchanged QOL-scores of a group may also contain individual scores that meaningfully changed (Crosby et al., 2003; Kemmler et al., 2010). Small changes on group level may be clinical significant, whereas on an individual level these changes do not reach clinical significance (Guyatt et al., 2002), since small score differences in individuals may be due to measurement errors if they do not exceed the range of variation. Moreover, not every single patient experiences the average score change of the group, but a stronger or weaker score-deviation. Thus, group comparisons are used for the evaluation of various treatments and health policy decisions, while for actual clinical decision making conclusions derived from the individual level should be used (Crosby et al., 2003). These methodological considerations illustrate how important it is to train clinicians and nursing staff in PRO-data interpretation, since a correct understanding of the data is the basis for adequate intervention.

\section{4 ePRO software example}

For obtaining the highest grade of feasibility and utility of PRO-assessments, the use of specialized software that fits perfectly the needs of both patients and clinicians is obligatory. In recent years some effort has been undertaken to develop and implement software solutions for QOL-assessment in clinical routine, for instance by Joerg Sigle (AnyQuest) and Galina Velikova and Irma Verdonck (OncoQuest). These tools use touch screens and have shown to be feasible in clinical practice. Implementation studies suggest that routine patient-monitoring using a software solution for assessment is beneficial for clinicians, patients and medical care in general (Wright et al., 2003; Velikova et al., 2004; Rogausch et al., 2009).

A further example for such a specific software solution is the computer-based health evaluation system (CHES). CHES has been especially been developed for electronic PRO- 
data capture and offers a variety of useful features for clinical routine and research purposes. Any required paper-pencil questionnaire can be implemented into the CHES program to facilitate all steps from data collection to result calculation, interpretation and statistical data analysis as well. CHES provides a database (e.g. MySQL or Oracle) for supplementary medical and psychosocial data. This database is particularly useful for research purposes, as it improves study logistics, reduces human resources and increases data quality. A so-called flag system enriches and facilitates the interpretation of QOL-data, since the implementation of reference values makes it possible to mark clinically relevant changes and problems. PRO-data can be accessed as a single assessment summary or as longitudinal graphical charts. The last one allows the immediate detection of changes and deficits in every single scale and gives an overview of the course of symptoms even if the clinician only takes a quick look at the graphs (Holzner et al., 2004). The availability of userfriendly and well-tested software for PRO-assessments is the key for successful routine implementation of PROs. CHES already offers a variety of beneficial features for PROapplication in clinical routine. A research project in cooperation with the EORCT Quality of Life Group (QLG) aims at the further development and distribution of CHES within the QLG for electronic QOL-assessment by means of the EORTC QLQ-C30 and its modules. The project comprises several steps of software development, multilingual software releases, a comprehensive manual and the work on additional features like computer-adaptive testing (CAT), telemonitoring and a web-forum for users and researchers.

Holzner et al. (2011) implemented CHES for routine QOL monitoring at a neuro-oncological outpatient unit and reported it to be feasible, as routinely conducted ePRO-assessments could be easily integrated into the schedule of the ward. The integration of QOL-data profiles into interdisciplinary team meetings offered the benefit that possible problem domains can be detected at an early stage and patient-centred care can be improved. The use of CHES for PRO-collection was also well accepted by another population of brain tumour patients (Erharter et al., 2010). On average patients needed only ten minutes to complete the EORTC QLQ-30 and the QLQ-BN20. Furthermore, repeated instrument administration was associated with a decrease of the average completion time. Clinicians reported the ePRO to be beneficial, as e.g. loss of bladder controlling would not have been detected adequately without ePRO.

\subsubsection{Patient example for PRO-profile}

To give an example for patient-reported symptom trajectories Figure 1 shows the PROprofiles of the male patient D.K. born in 1953. He attended the hospital due to first occurence of epileptic seizures in December 2009 where diagnostic procedures revealed an astrocytoma (WHO grade III, right side, parietotemporal). After confirmation through stereotactic biopsy chemotherapy with temozolomide started in January 2010. In March 2010, the patient was included in routine ePRO monitoring with EORTC QLQ-C30 and QLQ-BN20 at the neurooncological unit using the software CHES (see Figure 1).

From April 2010 until May 2010 the patient received additional whole-brain radiotherapy. After end of therapy, the patient was seen monthly at the outpatient unit for follow-up. Figure 1 shows the course of self-reported physical functioning and motor dysfunction from March 2010 until October 2010. During this period, the initially high symptom level decreased considerably, reaching average scores. Horizontal lines in the charts in Figure 1 show the $10^{\text {th }}$ percentile (red), the $25^{\text {th }}$ percentile (orange), and the $50^{\text {th }}$ percentile (green) from a reference group of neurooncological outpatients. Colouring of bars corresponds to 
these score ranges. Below each bar assessment date and abbreviation of treatment phase (chemotherapy (CT) and aftercare (AC)) is noted. Abbreviations on top of bars indicate specific interventions (pain intervention (PI) and psychooncological treatment $(\mathrm{PO})$ ).
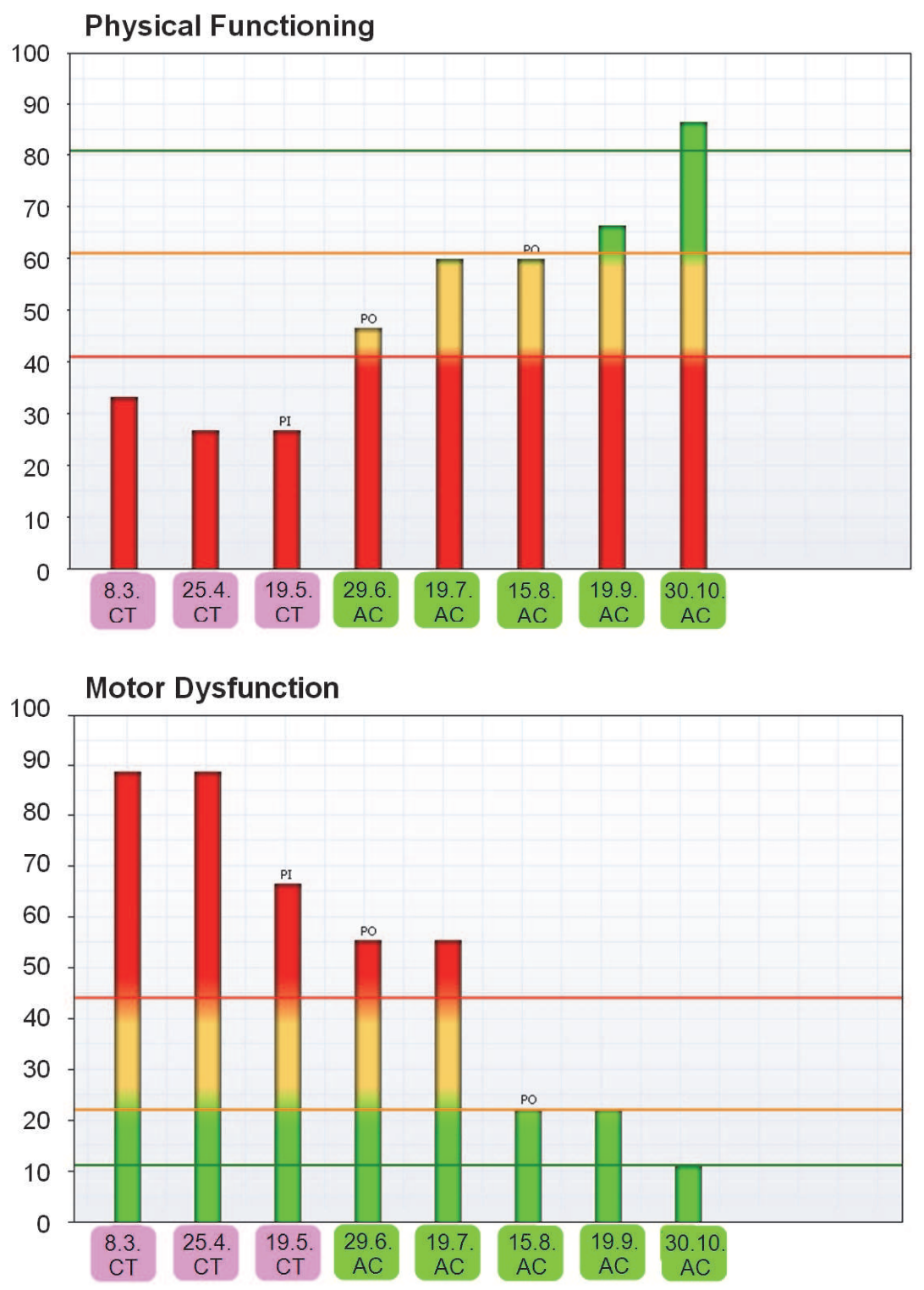

Fig. 1. Course of Physical Functioning (QLQ-C30) and Motor Dysfunction (QLQ-BN20) in a patient diagnosed with astrocytoma during chemotherapy and aftercare. 


\subsection{Home monitoring}

Even if QOL is regularly assessed within the hospital time schedule, information about patients' well-being is still incomplete, as their PRO-monitoring ends with hospital discharge. ePRO makes it possible to follow patients' in their home environment and to accompany them also during a phase, when hospital visits are not scheduled. Especially concerning chemotherapy, side-effects and treatment-related symptom burden are known to be most severe a few days after cytostatic drug application (Hawkins \& Grunberg, 2009). At that time, patients mostly are already back at home and are therefore left alone with their management of occurring adverse events. Perceived symptoms during times spent at home may be underestimated due to missing patient-monitoring. This gap may be overcome by ePROs conducted via phone or web-based assessments (telemonitoring). Telemonitoring in combination with alert systems enable clinicians to contact patients suffering from severe symptom burden and intervene appropriately even when he/she is not hospitalized (Balducci \& Stanta, 2000).

PRO-data from assessments in clinical routine can be merged with data from home monitoring and provide a database, which potentially enable clinicians to get a deeper insight in disease and treatment-related symptoms in general and to identify signs of adverse events at an early stage. In this way, prospective intervention can be provided to minimize complications. However, comprehensive and close-meshed tele monotoring of patients' QOL during their home stays requires additional administrative resources. Traditional paper-pencil questionnaires only have a low rate of return and are more expensive due to printing and postage costs. The internet provides an inexpensive and nowadays widespread technology that makes an easy, user friendly and reliable data collection possible. Though web-PROM imposes the need of a minimal computer literacy and internet use of patients, there is statistical data for Europe (Seybert \& Lööf, 2010) that supports the assumption that web-PROM may be feasible in a considerable percentage of cancer patients. In 2010, 70\% of European households had an internet connection and $69 \%$ of internet-users across all age groups used the internet regularly. The average rate of European internet-users who obtained health-related information via online sources doubled within the last six years (from 17\% in 2004 to $34 \%$ in 2010) and is supposed to increase further.

Although telemonitoring yet only reached an early stage of development, there are already a few studies available that document its use within oncological care. The most remarkable study was conducted by Bush et al. (2005) and collected QOL-data via web-PROM from patients who received hematopoietic stem cell transplantation. PRO-data was collected whether on a daily or monthly basis. Daily assessments consisted of only four out of 38 items, which varied according to a quasi-random frequency, and a global QOL item. Monthly assessments included all 38 items at once and needed to be completed within two successive days. Patients had to visit a website and log in with a private user name and a password to enter the online QOL-assessment. Overall compliance for both daily and monthly assessments was good and only three patients discontinued study participation due to their bad health condition. Therefore, data show that this way of PRO-data collection does not only include the less sick patients. Furthermore, high feasibility of web-PROM, high patient acceptance and user satisfaction war reported.

Telemonitoring takes patients' QOL outside the hospital setting into account and contributes to its integration into symptom estimation, monitoring and treatment. Especially for brain cancer patients, telemonitoring could be of particular benefit, as it may increase their feeling 
of security when they spend time at home. Eventually occurring symptoms are recognized by health care providers in real time despite the geographic distance between patient and medical services.

\subsection{Benefits from ePRO}

The traditional way of collecting PROs by means of paper-pencil questionnaires imposes several burdens to the patient, to clinical staff and financiers. Firstly missing data is more common in paper-pencil data collection as patients often may overlook an item and clinical staff is too busy to precisely control every single questionnaire. In addition, more human and time resources are needed, as questionnaires have to be printed in a sufficient number, tacked together, an stored so they can be handed over to patients, when needed. Subscales need to be calculated, put into an interpretable characteristic value and made accessible to clinicians. This procedure does not only gulp working time, costs for printing and staff, but is also vulnerable to missing data and errors in data transfer and calculation. ePROassessment by means of electronic devices like laptops and tablet PCs prevent these error sources and save time as well as costs. Admittedly, ePROs need some seed money to acquire technical equipment and software, but with regular use these devices amortize soon as printing of multi-paged questionnaires is no longer necessary and staff costs are reduced.

The implementation of PRO assessments of QOL or adverse events in clinical drug evaluation studies facilitates and accelerates data flow because intricate and defective data collection is cut short. Without PRO instruments clinicians ask patients about their symptoms and write a construed summary of these symptoms down in patients' charts, from which research assistants collect information and consign them into research data bases (Trotti et al., 2007). This process is highly endangered to lose and/or alter information given by patients' and needs much more human and time resources than the use of ePROassessments. By means of touch screen equipped tablet PCs patients can easily complete the PRO-assessment themselves. The possibility to fit font and button sizes to the needs of different patients groups allows that readability and handling of the assessment is practicable for e.g. elderly people as well (Giesinger et al., 2009a). As no computer literacy of patients is needed for PRO-completion, in principle all patients are able to participate in ePROs. They were shown to be well accepted by patients (Mullen et al., 2004; Velikova et al., 2010), valid (Abernethy et al., 2010) and equivalent to paper-pencil versions (Coons et al., 2009).

The advantage of time saving can also be applied to clinical routine. Admittedly, at the beginning of PRO implementation some time burden may be set on clinicians and nurses, as they need to be trained in software handling and result interpretation, but after a phase of familiarization PRO instruments contribute in time saving. Firstly, the completion of a PRO instrument encourages the patient to reflect more detailed on his/her health status and symptom burden and facilitates in that way the communication between patient and clinician/nurse about relevant problem areas. Secondly, via WiFi or LAN, patient's ePROdata is immediately accessible for the clinician, whose attention can be called to clinically relevant deteriorations or improvements. In fact, such an alert system helps clinicians to focus on issues important for the patient without long-winded enquiry of possible difficulties. Queue times of patients might meaningfully be padded by PRO-completion.

Furthermore, ePROs reduce staff costs, because score calculation, graphical illustration, alert systems and data storage are performed automatically. Time-saving aspects do not only concern data collection, but also identifying impaired QOL-domains. The implementation of 
reference levels simplifies the comparison between patient-groups or patients and normal population and in this way the detection of problems in QOL. Automatically generated reports may comprise only affected scales, the complete assessment or trajectories over time, depending on which information the user is interested in. Due to the instantly performed data calculation, clinicians directly can be informed after PRO completion about clinically remarkable PRO data that need adequate intervention. Clinicians may therefore pick these notable domains and address them to the patient without the need to undertake a longwinded exploration. In doing so, time can more effectively be spent on treatment and patient management, than on preliminary inquiry. The illustration of results by means of eye-catching and meaningful coloured graphs makes interpretation comfortable for both clinician and patient. Software solutions that include a storage database also simplify longterm data storage and backup. ePROs enhance data quality, since missing data is prevented by forwarding only to the remaining questions when all prior items were answered.

One common criticism to PROs is that patients are forced to answer annoying questions, which might be irrelevant to them. The development of CAT eradicates this flaw and makes ePRO-assessments for patients even more comfortable. CAT generates a patient-tailored question set and chooses appropriate items based on preceding answers. Information about diagnosis, progress of disease and treatment can also be considered for question compilation (Snyder et al., 2010).

Although many advantages of PRO assessments can be communicated, there are still popular counter-arguments rampant. Even if PRO-data is available many clinicians do not pay attention to them because of lack of time, human resources and an adequate PROinstrument and the assumption that directly from patients obtained information does not add any additional value (Luckett et al., 2009). Furthermore some clinicians argue that information on QOL are not of same importance as treatment decisions (Morris et al., 1998), equality of PRO instruments is doubtful (Barlesi et al., 2006) and the methodology of PRO measurement seems to be dubious. These objections can be devitalised, though. Meanwhile a broad variety of internationally validated and widely used PRO instruments is available (especially for cancer populations the forgoing mentioned EORTC QLQ-C30 and the FACT$\mathrm{G}$ with their supplemental modules). The use of PRO-instruments does usually not or only a few minutes prolong the clinical appointment (Frost et al., 2007). Particularly the implementation of ePROs solves the problem of time constraints as data is directly entered by patients, scores are calculated automatically and immediate information processing is possible. Electronic data capture was shown to need less time for instrument completion than paper-pencil versions (Drummond et al., 1995; Velikova et al., 1999; Taenzer et al., 2000). Table 2 summarizes pros and cons of PRO-assessments and illustrates, which benefits might outweigh commonly cited disadvantages of PRO-assessments.

Some studies illustrate that in clinical routine the usage of PROs offers further benefits than only time and cost saving. Based on PRO-data clinicians adjusted the dosage of analgesics in a more sophisticated way than without PRO-data (Trowbridge et al., 1997). The patientclinician communication was improved (Taenzer et al., 2000; Detmar et al., 2002a) and enhanced concerning discussed domains (Detmar et al., 2002a), if clinicians took advantage of provided QOL-data. Furthermore patients felt higher emotional support, clinicians became more sensitive for normally underestimated QOL-domains (Detmar et al., 2002a), patients were more satisfied with care and the building of a relationship to the clinician (Velikova et al., 2010) and reported a better QOL (Velikova et al., 2004). 
direct assessment of patients' perspective additional burden for patients

without intermediary (e.g. clinicians, proxies)

individually tailored therapy approaches in regard to PRO-data, targeted intervention

(medical, psychological)

improvement of patient-clinician communication

detection of unobvious problem domains (e.g. social domains, fatigue, sexual issues)

heightened awareness of clinicians for unobvious problem areas

patients' support in clinical decision making

longitudinal PRO-data capture allows to track the impact of medical intervention

ePRO: provide automated results calculation additional resources necessary (time, human and graphical presentation and financial resources)

time-saving assessment of relevant problem domains that need special attention

large variety of validated PRO-instruments dubious comparability of PRO-instruments (generic and specified)

CAT (computer adaptive testing) makes it possible to fit PROs to the patients' needs current, static PRO-instruments include also items irrelevant to a patients' current health status

Table 2. Advantages and disadvantages of PRO-assessments

\section{Conclusion}

The raising recognition of the patients' perspective in clinical research, drug evaluation and label claim issues demonstrate the importance of PRO-assessments. However, PROs could be used in more clinical settings than they are now implemented. Especially in clinical 
routine PRO-data have a variety of benefits to offer, since already their mere administration potentially has positive effects on patients and clinicians.

Recapitulating, the practical implications of this chapter are the following: The use of PROs within clinical routine is easily realizable since validated and reliable instruments are available. The conduction of PRO-assessments is further facilitated by effortlessly manageable software solutions. The participation in PROs encourages the patient to reflect detailed on different domains of their QOL even before the appointment with the clinician. Immediately transferred PRO-data enables the clinician to purposefully guide the conversation towards issues the patients reported to be important. In this way, time resources are optimal utilized: Queuing time is reasonably spent, because the patient gets prepared for the visit and within the appointment the clinician can spend more time on intervention and treatment. PROs allow therefore a targeted patient-clinician communication and a comprehensive as well as systematically performed screening for symptoms and impairments.

Concerning the feasibility of PRO-assessments within the schedule of a busy oncology clinic, ePROs and constantly ongoing software development support the regular use of these measures also with neurooncological patients. It is of crucial importance to fit available PRO-instruments and their electronic administration to the needs of health care providers' daily procedures. The smooth and effective implementation of PROs in clinical routine makes it more likely that they are accepted and supported by clinicians and nurses. Although the acceptance of PROs by patients is of major importance, the health care providers' attitude towards QOL-measurement should not be disregarded. Clinicians' contribution to PRO-assessment and PRO-data use influences substantially the success and effectiveness of PROs. The way how clinicians introduce PROs to patients may influence their feelings about them as well as their willingness to contribute. If patients are approached with a positive attitude they might experience PROs as an additional benefit instead of a further burden. Therefore, health care providers need to be directly approached to actively participate in QOL-measurement in the neurooncological setting. Even if patients are no longer able to provide self-reports, as it might especially occur in neurooncological patients, it is useful to ask significant others to substitutionally rate patients' QOL to continue the assessment of the patients' perception for symptom management.

Especially in neurooncological patients, whose median survival expectancy is rather low, it is of particular importance to assess QOL in order to be able to quickly intervene if impairments occur. The routine use of PRO-instruments allows a constant patient monitoring with little effort, which nevertheless makes individualized treatment possible. That patient-centred care is highly needed is demonstrated by study results of Bosma et al. (2009). They report that glioma patients differ in their QOL-patterns in regard to their survival time. Patients with a shorter survival (death within one year from diagnosis) were reported to have more general health problems during the course of disease and an increase of weakness of legs until four months after baseline. On the other hand, long-term survivors (still alive two years after diagnosis) had fewer general health problems, showed improvements of physical, social and emotional functioning and had stable or even decreasing brain specific symptom levels. The limited survival of most brain cancer patients requires a special focus on QOL, since curative treatment is unlikely and other aspects of life become more important.

Methodologically, there is still the need to establish reference scores for brain cancer patients with different diagnoses undergoing diverse therapies. Furthermore, there is a lack of 
randomized-controlled trials that investigate the effect of ePRO-based, targeted interventions in clinical routine. Future studies should put a focus on the evaluation of the precise impact of ePROs on medical care. A comprehensive knowledge about the impact of ePROs on the patient-clinician communication and on the provision of medical and psychosocial interventions would facilitate the patient-centred care and could therefore contribute to QOL improvement.

\section{Acknowledgement}

The work of Lisa M. Wintner was financially supported by a scholarship of the LeopoldFranzens-University Innsbruck, Austria.

\section{References}

Aaronson, N.K. Ahmedzai, S. Bergman, B. Bullinger, M. Cull, A. Duez, N.J. Filiberti, A. Flechtner, H. Fleishman, S.B. \& de Haes, J.C. (1993). The European Organization for Research and Treatment of Cancer QLQ-C30: a quality-of-life instrument for use in international clinical trials in oncology. J Natl Cancer Inst, Vol. 85, No. 5, pp. 365-76

Abernethy, A.P. Zafar, S.Y. Uronis, H. Wheeler, J.L. Coan, A. Rowe, K. Shelby, R.A. Fowler, R. \& Herndon, J.E., 2nd. (2010). Validation of the Patient Care Monitor (Version 2.0): a review of system assessment instrument for cancer patients. J Pain Symptom Manage, Vol. 40, No. 4, (Oct), pp. 545-58

Addington-Hall, J. \& Kalra, L. (2001). Who should measure quality of life? BMJ, Vol. 322, No. 7299, (Jun 9), pp. 1417-20

Arora, N.K. (2003). Interacting with cancer patients: the significance of physicians' communication behavior. Soc Sci Med, Vol. 57, No. 5, (Sep), pp. 791-806

Balducci, L. \& Stanta, G. (2000). Cancer in the frail patient. A coming epidemic. Hematol Oncol Clin North Am, Vol. 14, No. 1, (Feb), pp. 235-50, xi

Barlesi, F. Tchouhadjian, C. Doddoli, C. Astoul, P. Thomas, P. \& Auquier, P. (2006). [Quality of life: attitudes and perspectives of doctors in a thoracic oncology regional care network]. Sante Publique, Vol. 18, No. 3, (Sep), pp. 429-42

Basch, E. (2010). The missing voice of patients in drug-safety reporting. N Engl J Med, Vol. 362, No. 10, (Mar 11), pp. 865-9

Basch, E. Iasonos, A. Barz, A. Culkin, A. Kris, M.G. Artz, D. Fearn, P. Speakman, J. Farquhar, R. Scher, H.I. McCabe, M. \& Schrag, D. (2007). Long-term toxicity monitoring via electronic patient-reported outcomes in patients receiving chemotherapy. J Clin Oncol, Vol. 25, No. 34, (Dec 1), pp. 5374-80

Bosma, I. Reijneveld, J.C. Douw, L. Vos, M.J. Postma, T.J. Aaronson, N.K. Muller, M. Vandertop, W.P. Slotman, B.J. Taphoorn, M.J. Heimans, J.J. \& Klein, M. (2009). Health-related quality of life of long-term high-grade glioma survivors. Neuro Oncol, Vol. 11, No. 1, (Feb), pp. 51-8

Bossola, M. Murri, R. Onder, G. Turriziani, A. Fantoni, M. \& Padua, L. (2010). Physicians' knowledge of health-related quality of life and perception of its importance in daily clinical practice. Health Qual Life Outcomes, Vol. 8, No. pp. 43

Brown, P. Decker, P. Rummans, T. Clark, M. Frost, M. Ballman, K. Arusell, R. \& Buckner, J. (2008). A prospective study of quality of life in adults with newly diagnosed high- 
grade gliomas: comparison of patient and caregiver ratings of quality of life. Am J Clin Oncol, Vol. 31, No. 2, pp. 163-168

Bush, -.N. Donaldson, -.G. Moinpour, -.C. Haberman, -.M. Milliken, -.D. Markle, -.V. \& Lauson, -.J. (2005). Development, feasibility and compliance of a web-based system for very frequent QOL and symptom home self-assessment after hematopoietic stem cell transplantation. Qual Life Res, Vol. 14, No. 7, pp. 77-93

Campos, S. Davey, P. Hird, A. Pressnail, B. Bilbao, J. Aviv, R.I. Symons, S. Pirouzmand, F. Sinclair, E. Culleton, S. Desa, E. Goh, P. \& Chow, E. (2009). Brain metastasis from an unknown primary, or primary brain tumour? A diagnostic dilemma. Curr Oncol, Vol. 16, No. 1, (Jan), pp. 62-6

Carlson, L.E. Speca, M. Hagen, N. \& Taenzer, P. (2001). Computerized quality-of-life screening in a cancer pain clinic. J Palliat Care, Vol. 17, No. 1, pp. 46-52

Cella, D. \& Nowinski, C., J. (2002). Measuring quality of life in chronic illness: the functional assessment of chronic illness therapy measurement system. Arch Phys Med Rehabil, Vol. 83, No. 12 Suppl 2, pp. S10-7

Cella, D.F. Tulsky, D.S. Gray, G. Sarafian, B. Linn, E. Bonomi, A. Silberman, M. Yellen, S.B. Winicour, P. Brannon, J. \& et al. (1993). The Functional Assessment of Cancer Therapy scale: development and validation of the general measure. J Clin Oncol, Vol. 11, No. 3, (Mar), pp. 570-9

Coons, S. Gwaltney, C. Hays, R. Lundy, J. Sloan, J. Revicki, D. Lenderking, W. Cella, D. Basch, E. \& Force, I.e.T. (2009). Recommendations on evidence needed to support measurement equivalence between electronic and paper-based patient-reported outcome (PRO) measures: ISPOR ePRO Good Research Practices Task Force report. Value Health, Vol. 12, No. 4, pp. 419-429

Crosby, R.D. Kolotkin, R.L. \& Williams, G.R. (2003). Defining clinically meaningful change in health-related quality of life. J Clin Epidemiol, Vol. 56, No. 5, (May), pp. 395-407

Detmar, S.B. Aaronson, N.K. Wever, L.D. Muller, M. \& Schornagel, J.H. (2000). How are you feeling? Who wants to know? Patients' and oncologists' preferences for discussing health-related quality-of-life issues. J Clin Oncol, Vol. 18, No. 18, (Sep 15), pp. 3295301

Detmar, S.B. Muller, M.J. Schornagel, J.H. Wever, L.D.V. \& Aaronson, N.K. (2002a). Healthrelated quality-of-life assessments and patient-physician communication: a randomized controlled trial. JAMA, Vol. 288, No. 23, pp. 3027-34

Detmar, S.B. Muller, M.J. Schornagel, J.H. Wever, L.D.V. \& Aaronson, N.K. (2002b). Role of health-related quality of life in palliative chemotherapy treatment decisions. J Clin Oncol, Vol. 20, No. 4, pp. 1056-62

Drummond, H.E. Ghosh, S. Ferguson, A. Brackenridge, D. \& Tiplady, B. (1995). Electronic quality of life questionnaires: a comparison of pen-based electronic questionnaires with conventional paper in a gastrointestinal study. Qual Life Res, Vol. 4, No. 1, (Feb), pp. 21-6

Drury, M. Yudkin, P. Harcourt, J. Fitzpatrick, R. Jones, L. Alcock, C. \& Minton, M. (2000). Patients with cancer holding their own records: a randomised controlled trial. $\mathrm{Br} \mathrm{J}$ Gen Pract, Vol. 50, No. 451, pp. 105-10

Dubois, D. Dhawan, R. van de Velde, H. Esseltine, D. Gupta, S. Viala, M. \& de la Loge, C. (2006). Descriptive and prognostic value of patient-reported outcomes: the bortezomib experience in relapsed and refractory multiple myeloma. J Clin Oncol, Vol. 24, No. 6, pp. 976-82 
Farivar, S.S. Liu, H. \& Hays, R.D. (2004). Half standard deviation estimate of the minimally important difference in HRQOL scores? Expert Rev Pharmacoecon Outcomes Res, Vol. 4, No. 5, (Oct), pp. 515-23

Ferlay J, Shin HR, Bray F, Forman D, Mathers C and Parkin DM. GLOBOCAN 2008, Cancer Incidence and Mortality Worldwide: IARC CancerBase No. 10 [Internet]. Lyon, France: International Agency for Research on Cancer; 2010. Available from: http://globocan.iarc.fr

Frost, M. Bonomi, A. Cappelleri, J. Schunemann, H. Moynihan, T. \& Aaronson, N. (2007). Applying quality-of-life data formally and systematically into clinical practice. Mayo Clin Proc, Vol. 82, No. 10, pp. 1214-28

Ganz, P.A. \& Goodwin, P.J. (2007). Health-related quality of life measurement in symptom management trials. J Natl Cancer Inst Monogr, Vol. No. 37, pp. 47-52

Ganz, P.A. Moinpour, C.M. Pauler, D.K. Kornblith, A.B. Gaynor, E.R. Balcerzak, S.P. Gatti, G.S. Erba, H.P. McCoy, S. Press, O.W. \& Fisher, R.I. (2003). Health status and quality of life in patients with early-stage Hodgkin's disease treated on Southwest Oncology Group Study 9133. J Clin Oncol, Vol. 21, No. 18, (Sep 15), pp. 3512-9

Giesinger, J. Kemmler, G. Meraner, V. Gamper, E.M. Oberguggenberger, A. SpernerUnterweger, B. \& Holzner, B. (2009a). Towards the Implementation of Quality of Life Monitoring in Daily Clinical Routine: Methodological Issues and Clinical Implication. Breast Care (Basel), Vol. 4, No. 3, pp. 148-154

Giesinger, J.M. Golser, M. Erharter, A. Kemmler, G. Schauer-Maurer, G. Stockhammer, G. Muigg, A. Hutterer, M. Rumpold, G. \& Holzner, B. (2009b). Do neurooncological patients and their significant others agree on quality of life ratings? Health Qual Life Outcomes, Vol. 7, No. pp. 87

Guyatt, G.H. Osoba, D. Wu, A.W. Wyrwich, K.W. \& Norman, G.R. (2002). Methods to explain the clinical significance of health status measures. Mayo Clin Proc, Vol. 77, No. 4, (Apr), pp. 371-83

Hahn, C. \& Dunn, R. (2003). Prospective study of neuropsychologic testing and quality of life assessment of adults with primary malignant brain tumors. International Journal of Radiation, Oncology, Biology, Physica, Vol. 55, No. 4, pp. $992-999$

Hawkins, R. \& Grunberg, S. (2009). Chemotherapy-induced nausea and vomiting: challenges and opportunities for improved patient outcomes. Clin J Oncol Nurs, Vol. 13, No. 1, (Feb), pp. 54-64

Heimans, J. \& Taphoorn, M.J. (2002). Impact of brain tumour treatment on quality of life. J Neurol, Vol. 249, No. 8, pp. 955-60

Holzner, B. Schauer-Maurer, G. Stockhammer, G. Muigg, A. Hutterer, M. \& J., G. (2011). Computergestütztes Patient-reported Outcome Monitoring in der Neuroonkologie: Lebensqualität und Rezidiv beim Glioblastom. Wien Med Wochenschr, Vol. 161, No. 1-2, pp. 13-19

Holzner, B. Zabernigg, A. Kemmler, G. Baier, S. Kopp, M. \& Sperner Unterweger, B. (2004). Computerized assessment of quality of life in patients undergoing chemotherapy. Qual Life Res, Vol. 13, No. 9, pp. 1523

Janda, M. Steginga, S. Langbecker, D. Dunn, J. Walker, D. \& Eakin, E. (2007). Quality of life among patients with a brain tumor and their carers. J Psychosom Res, Vol. 63, No. 6, (Dec), pp. 617-23

Kemmler, G. Zabernigg, A. Gattringer, K. Rumpold, G. Giesinger, J. Sperner-Unterweger, B. \& Holzner, B. (2010). A new approach to combining clinical relevance and 
statistical significance for evaluation of quality of life changes in the individual patient. J Clin Epidemiol, Vol. 63, No. 2, (Feb), pp. 171-9

Luckett, T. Butow, P.N. \& King, M.T. (2009). Improving patient outcomes through the routine use of patient-reported data in cancer clinics: future directions. Psychooncology, Vol. 18, No. 11, (Nov), pp. 1129-38

Marino, P. Roche, H. Biron, P. Janvier, M. Spaeth, D. Fabbro, M. Linassier, C. Delozier, T. Martin, A.L. Santin, G. \& Moatti, J.P. (2008). Deterioration of quality of life of highrisk breast cancer patients treated with high-dose chemotherapy: the PEGASE 01 Quality of Life Study. Value Health, Vol. 11, No. 4, (Jul-Aug), pp. 709-18

McLachlan, S.A. Allenby, A. Matthews, J. Wirth, A. Kissane, D. Bishop, M. Beresford, J. \& Zalcberg, J. (2001). Randomized trial of coordinated psychosocial interventions based on patient self-assessments versus standard care to improve the psychosocial functioning of patients with cancer. J Clin Oncol, Vol. 19, No. 21, pp. 4117 - 25

Meraner, V. Giesinger, J. Kemmler, G. Taucher, S. Hubalek, M. Weber, B. Rumpold, G. Sperner-Unterweger, B. \& Holzner, B. (2009). Development of a screening tool for the identification of psychooncological treatment needs in breast cancer patients. Psychooncology, Vol. epub 13 Jan 2009, No. pp.

Morris, J. Perez, D. \& McNoe, B. (1998). The use of quality of life data in clinical practice. Qual Life Res, Vol. 7, No. 1, pp. 85 - 91

Mullen, K.H. Berry, D.L. \& Zierler, B.K. (2004). Computerized symptom and quality-of-life assessment for patients with cancer part II: acceptability and usability. Oncol Nurs Forum, Vol. 31, No. 5, (Sep), pp. E84-9

Nickolov, A. Beumont, J. Victorson, D. Peterman, A. Cella, D. Liepa, A. \& HA, F. (2005). Validation of functional assessment of cancer therapy: brain (FACT-Br) questionnaire and FACT-Br symptom index (FBrSI) in patients with recurrent highgrade glioma. . Paper presented at: Chicago Supportive Oncology Conference, 2005, ; Chicago, IL., Vol. No. (October 6-8), pp.

Osoba, D. (2007). Translating the science of patient-reported outcomes assessment into clinical practice. J Natl Cancer Inst Monogr, Vol. No. 37, pp. 5-11

Osoba, D. Brada, M. Prados, M.D. \& Yung, W.K. (2000). Effect of disease burden on healthrelated quality of life in patients with malignant gliomas. Neuro Oncol, Vol. 2, No. 4, pp. 221-8

Patrick, D.L. Burke, L.B. Powers, J.H. Scott, J.A. Rock, E.P. Dawisha, S. O'Neill, R. \& Kennedy, D.L. (2007). Patient-reported outcomes to support medical product labeling claims: FDA perspective. Value Health, Vol. 10 Suppl 2, No. (Nov-Dec), pp. S125-37

Press, O.W. LeBlanc, M. Lichter, A.S. Grogan, T.M. Unger, J.M. Wasserman, T.H. Gaynor, E.R. Peterson, B.A. Miller, T.P. \& Fisher, R.I. (2001). Phase III randomized intergroup trial of subtotal lymphoid irradiation versus doxorubicin, vinblastine, and subtotal lymphoid irradiation for stage IA to IIA Hodgkin's disease. J Clin Oncol, Vol. 19, No. 22, (Nov 15), pp. 4238-44

Reardon, D.A. \& Wen, P.Y. (2006). Therapeutic advances in the treatment of glioblastoma: rationale and potential role of targeted agents. Oncologist, Vol. 11, No. 2, (Feb), pp. 152-64

Revicki, D. Hays, R. Cella, D. \& Sloan, J. (2008). Recommended methods for determining responsiveness and minimally important differences for patient-reported outcomes. J Clin Epidemiol, Vol. 61, No. 2, pp. 102-109 
Rodriguez, K.L. Bayliss, N. Alexander, S.C. Jeffreys, A.S. Olsen, M.K. Pollak, K.I. Kennifer, S.L. Tulsky, J.A. \& Arnold, R.M. (2010). How oncologists and their patients with advanced cancer communicate about health-related quality of life. Psychooncology, Vol. 19, No. 5, (May), pp. 490-9

Rogausch, A. Sigle, J. Seibert, A. Thuring, S. Kochen, M.M. \& Himmel, W. (2009). Feasibility and acceptance of electronic quality of life assessment in general practice: an implementation study. Health Qual Life Outcomes, Vol. 7, No. pp. 51

Rosenbloom, S.K. Victorson, D.E. Hahn, E.A. Peterman, A.H. \& Cella, D. (2007). Assessment is not enough: a randomized controlled trial of the effects of HRQL assessment on quality of life and satisfaction in oncology clinical practice. Psychooncology, Vol. 16, No. 12, (Dec), pp. 1069-79

Rothman, M.L. Beltran, P. Cappelleri, J.C. Lipscomb, J. \& Teschendorf, B. (2007). Patientreported outcomes: conceptual issues. Value Health, Vol. 10 Suppl 2, No. (Nov-Dec), pp. S66-75

Sanchez, R. Ballesteros, M. \& Arnold, B.J. (2011). Validation of the FACT-G scale for evaluating quality of life in cancer patients in Colombia. Qual Life Res, Vol. 20, No. 1, (Feb), pp. 19-29

Seybert, H. \& Lööf, A.(2010). Internet usage in 2010 - Households and Individuals, Availble from: http://epp.eurostat.ec.europa.eu/cache/ITY_OFFPUB/KS-QA-10050/EN/KS-QA-10-050-EN.PDF

Sizoo, E.M. Braam, L. Postma, T.J. Pasman, H.R. Heimans, J.J. Klein, M. Reijneveld, J.C. \& Taphoorn, M.J. (2010). Symptoms and problems in the end-of-life phase of highgrade glioma patients. Neuro Oncol, Vol. 12, No. 11, (Nov), pp. 1162-6

Sneeuw, K.C. Aaronson, N.K. Osoba, D. Muller, M.J. Hsu, M.A. Yung, W.K. Brada, M. \& Newlands, E.S. (1997). The use of significant others as proxy raters of the quality of life of patients with brain cancer. Med Care, Vol. 35, No. 5, pp. 490-506

Snyder, C.F. Jensen, R.E. Geller, G. Carducci, M.A. \& Wu, A.W. (2010). Relevant content for a patient-reported outcomes questionnaire for use in oncology clinical practice: Putting doctors and patients on the same page. Qual Life Res, Vol. 19, No. 7, (Sep), pp. 1045-55

Sprangers, M.A. Cull, A. Groenvold, M. Bjordal, K. Blazeby, J. \& Aaronson, N.K. (1998). The European Organization for Research and Treatment of Cancer approach to developing questionnaire modules: an update and overview. EORTC Quality of Life Study Group. Qual Life Res, Vol. 7, No. 4, pp. 291-300

Stupp, R. Mason, W. van den Bent, M. Weller, M. Fisher, B. Taphoorn, M. Belanger, K. Brandes, A. Marosi, C. Bogdahn, U. Curschmann, J. Janzer, R. Ludwin, S. Gorlia, T. Allgeier, A. Lacombe, D. Cairncross, J. Eisenhauer, E. Mirimanoff, R. European Organisation for Research and Treatment of Cancer Brain Tumor and Radiotherapy Groups \& National Cancer Institute of Canada Clinical Trials Group. (2005). Radiotherapy plus concomitant and adjuvant temozolomide for glioblastoma. $N$ Engl J Med, Vol. 352, No. 10, pp. 987-996

Taenzer, P. Bultz, B.D. Carlson, L.E. Speca, M. DeGagne, T. Olson, K. Doll, R. \& Rosberger, Z. (2000). Impact of computerized quality of life screening on physician behaviour and patient satisfaction in lung cancer outpatients. Psychooncology, Vol. 9, No. 3, pp. 203-13

Taphoorn, M. Stupp, R. Coens, C. Osoba, D. Kortmann, R. van den Bent, M. Mason, W. Mirimanoff, R. Baumert, B. Eisenhauer, E. Forsyth, P. \& Bottomley, A. (2005). 
Health-related quality of life in patients with glioblastoma: a randomised controlled trial. Lancet Oncol, Vol. 6, No. 12, pp. 937-944

Taphoorn, M.J. Sizoo, E.M. \& Bottomley, A. (2010). Review on quality of life issues in patients with primary brain tumors. Oncologist, Vol. 15, No. 6, pp. 618-26

Trotti, A. Colevas, A.D. Setser, A. \& Basch, E. (2007). Patient-reported outcomes and the evolution of adverse event reporting in oncology. J Clin Oncol, Vol. 25, No. 32, (Nov 10), pp. 5121-7

Trowbridge, R. Dugan, W. Jay, S.J. Littrell, D. Casebeer, L.L. Edgerton, S. Anderson, J. \& O'Toole, J.B. (1997). Determining the effectiveness of a clinical-practice intervention in improving the control of pain in outpatients with cancer. Acad Med, Vol. 72, No. 9, (Sep), pp. 798-800

U.S. Food and drug administration (FDA). (2006). Guidance for Industry. Patient-Reported Outcome Measures: Use in Medical Product Development to Support Labeling Claims. Federal Register, Vol. 74, No. 35, pp. 65132-133

Velikova, G. Booth, L. Smith, A.B. Brown, P.M. Lynch, P. Brown, J.M. \& Selby, P.J. (2004). Measuring quality of life in routine oncology practice improves communication and patient well-being: a randomized controlled trial. J Clin Oncol, Vol. 22, No. 4, (Feb 15), pp. 714-24

Velikova, G. Keding, A. Harley, C. Cocks, K. Booth, L. Smith, A.B. Wright, P. Selby, P.J. \& Brown, J.M. (2010). Patients report improvements in continuity of care when quality of life assessments are used routinely in oncology practice: secondary outcomes of a randomised controlled trial. Eur J Cancer, Vol. 46, No. 13, (Sep), pp. 2381-8

Velikova, G. Wright, E.P. Smith, A.B. Cull, A. Gould, A. Forman, D. Perren, T. Stead, M. Brown, J. \& Selby, P.J. (1999). Automated collection of quality-of-life data: a comparison of paper and computer touch-screen questionnaires. J Clin Oncol, Vol. 17, No. 3, pp. 998-1007

Walker, M. Brown, J. Brown, K. Gregor, A. Whittle, I. \& Grant, R. (2003). Practical problems with the collection and interpretation of serial quality of life assessment in patients with malignant glioma. J Neurooncol, Vol. 63, No. 2, pp. 179-186

Webster, K. Cella, D. \& Yost, K. (2003). The Functional Assessment of Chronic Illness Therapy (FACIT) Measurement System: properties, applications, and interpretation. Health Qual Life Outcomes, Vol. 1, No. pp. 79

Wright, E.P. Selby, P.J. Crawford, M. Gillibrand, A. Johnston, C. Perren, T.J. Rush, R. Smith, A. Velikova, G. Watson, K. Gould, A. \& Cull, A. (2003). Feasibility and compliance of automated measurement of quality of life in oncology practice. J Clin Oncol, Vol. 21, No. 2, (Jan 15), pp. 374-82

Zabora, J. Brintzenhofeszoc, K. Curbow, B. Hooker, C. \& Piantadosi, S. (2001). The prevalence of psychological distress by cancer site. Psychooncology, Vol. 10, No. 1, (Jan-Feb), pp. 19-28

Zigmond, A. \& Snaith, R. (1983). The hospital anxiety and depression scale. Acta Psychiatr Scand, Vol. 67, No. 6, pp. 361-370 


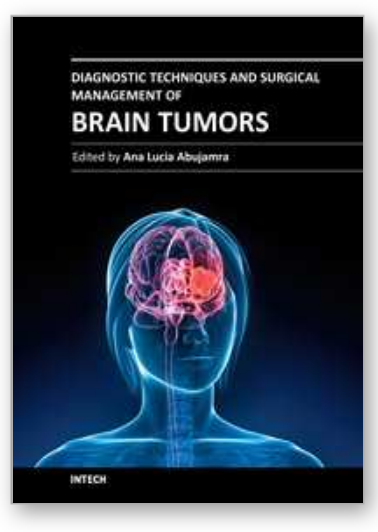

\author{
Diagnostic Techniques and Surgical Management of Brain Tumors \\ Edited by Dr. Ana Lucia Abujamra
}

ISBN 978-953-307-589-1

Hard cover, 544 pages

Publisher InTech

Published online 22, September, 2011

Published in print edition September, 2011

The focus of the book Diagnostic Techniques and Surgical Management of Brain Tumors is on describing the established and newly-arising techniques to diagnose central nervous system tumors, with a special focus on neuroimaging, followed by a discussion on the neurosurgical guidelines and techniques to manage and treat this disease. Each chapter in the Diagnostic Techniques and Surgical Management of Brain Tumors is authored by international experts with extensive experience in the areas covered.

\title{
How to reference
}

In order to correctly reference this scholarly work, feel free to copy and paste the following:

Lisa M. Wintner, Johannes M. Giesinger and Bernhard Holzner (2011). Patient-Reported Outcome Monitoring in Brain Tumour Patients: Benefits and Requirements, Diagnostic Techniques and Surgical Management of Brain Tumors, Dr. Ana Lucia Abujamra (Ed.), ISBN: 978-953-307-589-1, InTech, Available from:

$\mathrm{http}: / / w w w . i n t e c h o p e n . c o m / b o o k s / d i a g n o s t i c-t e c h n i q u e s-a n d-s u r g i c a l-m a n a g e m e n t-o f-b r a i n-t u m o r s / p a t i e n t-$ reported-outcome-monitoring-in-brain-tumour-patients-benefits-and-requirements

\section{INTECH}

open science | open minds

\section{InTech Europe}

University Campus STeP Ri

Slavka Krautzeka 83/A

51000 Rijeka, Croatia

Phone: +385 (51) 770447

Fax: +385 (51) 686166

www.intechopen.com

\section{InTech China}

Unit 405, Office Block, Hotel Equatorial Shanghai

No.65, Yan An Road (West), Shanghai, 200040, China

中国上海市延安西路65号上海国际贵都大饭店办公楼 405 单元

Phone: +86-21-62489820

Fax: +86-21-62489821 
(C) 2011 The Author(s). Licensee IntechOpen. This chapter is distributed under the terms of the Creative Commons Attribution-NonCommercialShareAlike-3.0 License, which permits use, distribution and reproduction for non-commercial purposes, provided the original is properly cited and derivative works building on this content are distributed under the same license. 